\title{
Novelty and Foreseeing Research Trends: The Case of Astrophysics and Astronomy
}

\author{
Attila Varga \\ University of Arizona, School of Sociology, P.O. Box 210027, Tucson, AZ 85721-0027, USA \\ Received 2017 October 17; revised 2017 December 29; accepted 2018 January 2; published 2018 May 11
}

\begin{abstract}
Metrics based on reference lists of research articles or on keywords have been used to predict citation impact. The concept behind such metrics is that original ideas stem from the reconfiguration of the structure of past knowledge, and therefore atypical combinations in the reference lists, keywords, or classification codes indicate future highimpact research. The current paper serves as an introduction to this line of research for astronomers and also addresses some of the methodological questions in this field of innovation studies. It is still not clear if the choice of particular indexes, such as references to journals, articles, or specific bibliometric classification codes affects the relationship between atypical combinations and citation impact. To understand more aspects of the innovation process, a new metric has been devised to measure to what extent researchers are able to anticipate the changing combinatorial trends of the future. Results show that the variant of the latter anticipation scores that is based on paper combinations is a good predictor of the future citation impact of scholarly works. The study also shows that the effects of tested indexes vary with the aggregation levels that were used to construct them. A detailed analysis of combinatorial novelty in the field reveals that certain sub-fields of astronomy and astrophysics have different roles in the reconfiguration of past knowledge.
\end{abstract}

Key words: publications, bibliography - sociology of astronomy

\section{Introduction}

It is important for faculties and funding agencies to be able to foresee the future research impact of individuals and research groups when hiring new faculty or awarding research funding (Clauset et al. 2017). A growing body of research is trying to understand how scientific research becomes impactful. In the past decade, data availability and computing power have allowed scholars to develop sophisticated measures for predicting the future citation impacts of papers, patents, and authors. The problem of predicting research impact can be approached in several ways. Some researchers have focused on individuals and have studied how career stage, past productivity, or institutional affiliation affect impact (e.g., Kurtz \& Henneken 2015, for a recent review see Clauset et al. 2017). Another line of inquiry aims to foresee the emergence of new and popular technologies and fields of research (Small et al. 2014).

This paper introduces and improves upon a fertile branch of these efforts that we may call "combinatorial innovation," which aims to theorize and model the process of innovation development and dissemination itself (Schilling \& Green 2011; Uzzi et al. 2013; Boyack \& Klavans 2014; Leahey \& Moody 2014; Kaplan \& Vakili 2015; Lee et al. 2015; Trapido 2015; Youn et al. 2015; for an extensive review see: Savino et al. 2017). We have three goals: (1) to evaluate various measurements of combinatorial novelty for citation predictions; (2) to introduce a combinatorial measure for the prognosis of scientific impact based on early anticipation of future trends; and (3) to contextualize the relevance of the diversity of combinatorial novelty measures in astronomy and astrophysics.

The main tenet of the paradigm of combinatorial innovation is that novel and original ideas combine past knowledge in a new way (Fleming 2001; Thagard 2012). A measure of combinatorial novelty generally indicates whether a combination of knowledge elements is typical or atypical given the past usage pattern of those elements. Despite the diversity of specific research focuses and methodologies, all studies found (mostly positive) an association between combinatorial novelty and citation impact, therefore this family of indicators could be utilized to identify trends in science. It must be emphasized that this particular approach to novelty measures the distance/ similarity of co-occurrences. This approach can be traced back to the early twentieth century, to Schumpeter's (1934) innovation theory and to Poincaré's (1910) philosophy of science. Poincare stressed that combinations are more innovative and radical if they combine disparate things. That assumption regarding the relationship between the distance/ dissimilarity of ideas and innovativeness still has an important role in this paradigm.

"Maximal marginal relevance" is a similar concept in information retrieval (Carbonell \& Goldstein 1998; Clarke et al. 2008, recently: An \& Huang 2017). It is a search result ranking criterion that assigns higher ranks to relevant records that contain newer content than previously ranked relevant records. A crucial difference, however, is that while combinatorial novelty evaluates the distance/dissimilarity of pairs of elements, this retrieval concept evaluates only the individual elements in the set. For instance, to borrow an example from Clarke et al. (2008), a search for "jaguar" gives a document that discusses jaguar as a car brand high novelty if the search until that point only retrieved hits about the jaguar as cat. A document that combines the topic of the car and the cat the first time, is not necessarily marginally novel. If all the information that such a document contains has already been covered by previous search hits about both the car and the animal, the document has low novelty. From the perspective of combinatorial innovation, however, it is an atypical combination and considered to be highly novel, because it is unusual to combine those elements.

Returning to the literature on combinatorial innovation, the contents of documents or the combined elements in research practice are typically measured as bibliometric classification 
codes assigned by indexing services, keywords, or journals. Although these studies share this basic insight and methodology, they are concerned with different aspects of the innovation process, sample vastly different fields of science and technology, and establish their measures of novelty through various systems of reference list aggregation or keywords. There are several discipline-specific bibliometric classification systems, and other classification systems that journals and citation indexing services assign to publications. One can also use cited journal pairs as building blocks of combinations. The choice of the classification system is sometimes influenced by theoretical considerations. For example, for studies of the citation impact of interdisciplinary research (Lariviere et al. 2015; Yegros-Yegros et al. 2015), and for organizational theorists (Leahey \& Moody 2014), atypical combinations are interesting because they span organizations and institutions. Therefore, combined elements must express organizational boundaries. However, the same theoretical construct can be operationalized as a classification system representing subfields (Leahey \& Moody 2014), or as Web of Science subject categories representing sub-disciplines (Lariviere et al. 2015; Yegros-Yegros et al. 2015). On several occasions the choice is not justified in detail (Uzzi et al. 2013; Lee et al. 2015).

The paper will test and compare different aggregation levels of reference list combinations to predict high-impact papers in astronomy, more specifically examining how the operationalized definition of combinatorial novelty affects its relationship with citation impact. The question of aggregation of scientometric indicators is in fact quite general, and it requires great caution and reflection for students in scientometrics (Leydesdorff 2001). There are two important dimensions of aggregation that will be investigated here: (1) time: past, present, future; and (2) classification levels: references to papers, to journals, to subdisciplines. Moreover, since the references to papers (the lowest classification level of ideas in the study) will be investigated herein, "absolute novelty" can also be defined in the context of this paper. While co-citations to journals and sub-fields endure for a long time, the co-citation of particular papers is frequently unprecedented.

The paper will present a diversity of combinatorial novelty indexes, and study their associations with citation impact, while aiming to filter out confounding factors. First, choosing a single discipline to study scientometric indicators, similar to Kurtz \& Henneken (2015), helps to filter out disciplinary effects. For example, Boyack \& Klavans (2014) showed that the results of Uzzi et al. (2013) on the effect of the novelty and conventionality of published science papers on citation impact was confounded by disciplinary differences. Studying astronomy in particular also helps to minimize the influence of industry-financed research on the novelty-seeking behavior of research groups. Evans (2010) argued that biochemistry, which is heavily sponsored by industrial companies, produces research that is less theory driven because the research is sponsored by industry. It is assumed that research in astronomy and astrophysics is primarily concerned with purely scientific questions, therefore the influence of corporate research is negligible. Second, the tested novelty indexes are constructed on three levels of aggregation: references to research documents (articles, or letters), referenced journals, and Web of Science subject categories. The latter is a journal classification system used extensively by researchers studying interdisciplinary science (Porter et al. 2007; Rafols \& Meyer 2010).
These three levels of reference aggregation can control for the extent of institutionalization from research articles to highly institutionalized sub-disciplines.

A new set of indexes of combinatorial novelty will also be introduced and tested along with the extant measures. If we assume that novel combinations are important, it is straightforward to hypothesize that anticipating the trends in the reconfiguration of science communication early on helps to disseminate new ideas. Aside from combinatorial novelty, it will be shown how combinations that are gaining popularity early on or are becoming popular now will, in the future, affect the citation impacts for publications.

This approach to novelty diverges from the concept introduced above. Instead of quantifying the atypicality of combinations, and attributing novelty to them, in this case the temporal shift of combinatorial activity is measured, and the theoretical assumption is that early trendsetting behavior is rewarded by the scientific community. Certainly it is not precluded that trendsetting is associated with novelty, but it is more straightforward to interpret the following measures as accurate anticipations in the shift in the focus of a research field. It is assumed that constructing these anticipation measures is another useful test of which level of analysis (articles, journals, and subject categories) is more predictive of future citation gain, and how the dynamics of these levels differ.

Although the study design is explorative and may seem technical, as demonstrated below, it will provide refutations to past theories of how new approaches and discoveries gain footing. This paper not only serves as an introduction to this exciting research agenda for the audience of $A p J S$, it also highlights how the studied intellectual and institutional factors play out in a specific discipline: astronomy/astrophysics. The final section of the paper will present "maps" of the core journals of astronomy and astrophysics as citation networks, where the behavior of the tested indexes can be investigated in a more concrete fashion. These maps can shed light on how different territories or clusters of publication venues drive trends at different institutional levels in the discipline. This presentation is very important because-given the narrow focus on a single coherent discipline-one can have a contextualized understanding of the meaning of the tested indexes.

\section{Tested Indexes and Data}

The samples of astronomy and astrophysics bibliographic records were retrieved from Web of Science. All the references are assigned to subject categories based on journal classification. The primary data set contains all publications (articles and letters) that fall under any physics-related subject category in the past few decades, additionally including the subject category "Multidisciplinary Sciences," which contains all journals that publish articles across the sciences (such as Science and Nature). To create the relevant sample from the records in the primary data set, all publications were included that cited at least two journals listed under the subject category "Astronomy and Astrophysics." This sampling procedure is inclusive; instead of sampling only the journals that are categorized as astronomy and astrophysics, several other physics publications were selected for analysis outside of strictly astronomical journals. This sampling approach reflects the general trend toward interdisciplinarity in physics 
Table 1

Novelty Indexes Tested in the Study

\begin{tabular}{|c|c|c|}
\hline Index & Formula & Description \\
\hline $\mathrm{JR}$ & $F_{T_{-1,0}}\left(\left\{J_{i} ; J_{j}\right\}\right) W^{\mathrm{a}}$ & $\begin{array}{l}\text { Atypical/novel journal combinations. The number of times journals } J_{i} \text { and } J_{j} \text { have been co-cited at } T_{-1} \text { and } T_{0} \text { weighted by the } \\
\text { normalization weight } W . W \text { is based on the past and present frequencies. }\end{array}$ \\
\hline SC & $F_{T-1,0}\left(\left\{S_{i} ; S_{j}\right\}\right) W^{\mathrm{a}}$ & $\begin{array}{l}\text { Atypical/novel subject category combinations. The number of times subject categories } S C_{i} \text { and } S C_{j} \text { have been co-cited at } T_{-1} \text { and } T_{0} \\
\text { weighted by the normalization weight } W . W \text { is based on the past and present frequencies. }\end{array}$ \\
\hline CIT & $F_{T_{-1,0}}\left(\left\{C_{i} ; C_{j}\right\}\right) W^{\mathrm{a}}$ & $\begin{array}{l}\text { Atypical/novel citation combinations. The number of times papers } C_{i} \text { and } C_{j} \text { have been co-cited at } T_{-1} \text { and } T_{0} \text { weighted by the } \\
\text { normalization weight } W . W \text { is based on the past and present frequencies. }\end{array}$ \\
\hline NCIT & $N_{T_{0}}\left(\left\{C_{i} ; C_{j}\right\}\right)$ & New combinations. From this binary variable the percentage of articles that have not been co-cited in the past has been calculated. \\
\hline
\end{tabular}

Note.

${ }^{\mathrm{a}} W=1 /\left(d_{i} d_{j}\right)$ is a normalization factor. $d_{i}$ stands for the number of times an article, journal, or subject category $i$ has been cited in the given time interval.

(Sinatra et al. 2015). For 2003 this procedure results in $\sim 17,000$ articles. The tests that are presented below are based on the 2003 data, and the Appendix shows key findings for 2007. To calculate past and future frequencies of combinations, and to derive citation impact, a 7-year window was used after and before the sampled year. The above sampling procedure was applied to create both of these windows. I will refer to these three time intervals throughout the paper as present $T_{0}$, past $T_{-1}$, and future $T_{1}$.

Relying on references to papers in order to construct an index of combinatorial novelty is unprecedented. One probable reason why it is such a neglected measurement choice is that it gives a large number of data points. Doing so, however, provides a fine-grained and dynamic picture of how communication is configured in science (Small 1978; Leydesdorff 1998). The reference list of a paper is $C=\left(C_{1}, C_{2} \ldots C_{n}\right)$, and a co-citation of two articles in this reference list is denoted as $\left\{C_{i} ; C_{j}\right\} \quad\left(C_{i} \in C, C_{j} \in C\right)$. From $C$ we can derive the aggregated reference lists at the journal and subject category level. Two combined journals and two combined subject categories are denoted as $\left\{J_{i} ; J_{j}\right\}$ and $\left\{S_{i} ; S_{j}\right\}$, respectively. One journal can be assigned to several subject categories, and in these cases all the subject categories were included in the analysis.

To construct an index of combinatorial novelty we must first measure the raw count of combinations. This gives a raw affinity score for the combinations. All these combination frequencies at the three levels were recorded in the three time intervals defined above (present $T_{0}$, past $T_{-1}$, and future $T_{1}$ ). Studies of combinatorial innovation create baseline frequencies based on the sum of past and present combinations. The past and future combinatorial frequencies are recorded to calculate the anticipation scores. These raw counts for the "present" are expressed as $F_{T_{0}}\left(\left\{C_{i} ; C_{j}\right\}\right)$ for citation pairs, $F_{T_{0}}\left(\left\{J_{i} ; J_{j}\right\}\right)$ for journal pairs, and $F_{T_{0}}\left(\left\{S_{i} ; S_{j}\right\}\right)$ for subject category pairs. Journals and subject categories exist for a longer time frame, and absolute novelty-when a pair is totally unprecedented in the past $\left(F_{T_{-1}}\left(\left\{J_{i} ; J_{j}\right\}\right)=0, F_{T_{-1}}\left(\left\{S_{i} ; S_{j}\right\}\right)=0\right)$-in the sampled time interval is rarer than new paper combinations. In this latter case absolute novelty is defined as:

$$
N_{T_{0}}\left(\left\{C_{i}, C_{j}\right\}\right)= \begin{cases}0, & F_{T_{-1}}\left(\left\{C_{i} ; C_{j}\right\}\right)>0 \\ 1, & F_{T_{-1}}\left(\left\{C_{i} ; C_{j}\right\}\right)=0 .\end{cases}
$$

Therefore, $N_{T_{0}}\left(\left\{C_{i}, C_{j}\right\}\right)$ is 1 if the articles $C_{i}$ and $C_{j}$ have never been cited together before, and zero otherwise. Note that this index disregards the degree of distance of the combined elements: two entities are either related or not.
The second component of a novelty score is a normalizing weight. This serves to deflate the raw counts of combined pairs if the citations to the individual constituents of the pair are higher. This is sometimes derived from a Monte Carlo algorithm (Uzzi et al. 2013), but is mostly defined as a simple function of the frequency of the individual combined elements and expressing an expected count of the pair (e.g., Leahey \& Moody 2014; Lee et al. 2015). Here, a simple weight $W=$ $1 /\left(d_{i} d_{j}\right)$ is defined, where $d_{i}$ and $d_{j}$ stands for the number of times the given articles $C_{i}$ and $C_{j}$, journals $J_{i}$ and $J_{j}$, or subject categories $S C_{i}$ and $S C_{j}$, have been cited individually in the relevant time interval.

Similar to the previously discussed studies, a normalized score expresses the novelty-or more specifically the atypicality - of journal (referred as JR) and subject category pairs (SC). (Table 1 summarizes these scores in a comprehensive way). The higher these scores are, the more "atypical" (or novel) the combination is. For these two scores the recorded time intervals include the past and present. For the raw cocitations to articles a normalized score is also created (CIT) in a similar fashion, which indicates if a combination is less expected given the popularity of combined papers in the past and present. All three scores pertain to combinations; to aggregate them to the level of referencing articles the mean of the scores was calculated. Uzzi et al. (2013) suggested that both novelty and conventionality are important to create a groundbreaking paper. These are two separate dimensions of a publication, and the central tendency of their combination novelty score distribution is used to measure conventionality, while novelty is defined as the tail of this distribution. To include this theory in the test, aside from the mean, the 90th percentile of the scores is also investigated below (the score of the most atypical pairs).

NCIT is the index of the percentage of completely new cocited article pairs $N_{T_{0}}\left(\left\{C_{i}, C_{j}\right\}\right)$. No weighting was applied to this index.

Finally, six new scores are introduced based on the concept of anticipating future trends in the changing configuration of combinations (Table 2). First, there are three scores that contrast the past usage of referencing behavior with their emerging future usage. To define anticipation scores for cocited articles (ACIT) I simply use the number of times the combination appears in the future, $F_{T_{1}}\left(\left\{J_{i} ; J_{j}\right\}\right)$, and normalize it with $W$. $W$ in this case is based on the past and present frequencies. Given how popular the cited papers were separately, it expresses how popular their combination becomes in the future, with a higher index indicating more popular cocitation in the future. The anticipation scores for journals and 
Table 2

Anticipation Indexes Tested in the Study

\begin{tabular}{|c|c|c|}
\hline Index & Formula & Description \\
\hline ACIT & $F_{T_{1}}\left(\left\{C_{i} ; C_{j}\right\}\right) W^{\mathrm{a}}$ & $\begin{array}{l}\text { Anticipation score for cited papers. The simple count of how many times co-cited papers appear together in the future } \\
\text { (at } T_{1} \text { ) normalized with } W \text {. } W \text { in this case is based on the past and present frequencies. }\end{array}$ \\
\hline AJR & $\frac{F_{T_{1}}\left(\left\{J_{i}, J_{j}\right\}\right)}{\sum_{i, j} F_{T_{1}}\left(\left\{J_{i} ; J_{j}\right\}\right)}-\frac{F_{T_{-1}}\left(\left\{J_{i} ; J_{j}\right\}\right)}{\sum_{i, j} F_{T_{-1}}\left(\left\{J_{i}, J_{j}\right\}\right)}$ & $\begin{array}{l}\text { Anticipation score for journals. The difference of the percentages between the past } T_{-1} \text { and future } T_{1} \text { frequencies of co- } \\
\text { cited journal pairs. }\end{array}$ \\
\hline ASC & $\frac{F_{T_{1}}\left(\left\{S_{;} S_{j}\right\}\right)}{\sum_{i, j} T_{T_{1}}\left(\left\{S_{i} ; S_{j}\right\}\right)}-\frac{F_{T_{-1}}\left(\left\{S_{i} ; S_{j}\right\}\right)}{\sum_{i, j} T_{T_{-1}}\left(\left\{S_{i} ; S_{j}\right\}\right)}$ & $\begin{array}{l}\text { Anticipation score for subject categories. The difference of the percentages between the past } T_{-1} \text { and future } T_{1} \\
\text { frequencies of co-cited subject category pairs. }\end{array}$ \\
\hline JR (alt.) & $\frac{F_{T_{0}}\left(\left\{J_{i}, J_{j}\right\}\right)}{F_{T_{-1}}\left(\left\{I_{i}, J_{j}\right\}\right)+1}$ & $\begin{array}{l}\text { Alternative novelty measure based on the concept of anticipation for journals. The ratio of the frequency of present and } \\
\text { past combinations of } J_{i} \text { and } J_{j} \text {. }\end{array}$ \\
\hline SC (alt.) & $\frac{F_{T_{0}}\left(\left\{S_{i}, S_{j}\right\}\right)}{F_{T_{-1}}\left(\left\{S_{i} ; S_{j}\right\}\right)+1}$ & $\begin{array}{l}\text { Alternative novelty measure based on the concept of anticipation for subject categories. The ratio of the frequency of } \\
\text { present and past combinations of } S_{i} \text { and } S_{j} \text {. }\end{array}$ \\
\hline CIT (alt.) & $\frac{F_{T_{0}}\left(\left\{C_{i} ; C_{j}\right\}\right)}{F_{T_{-1}}\left(\left\{C_{i} ; C_{j}\right\}\right)+1}$ & $\begin{array}{l}\text { Alternative novelty measure based on the concept of anticipation for citations. The ratio of the frequency of present and } \\
\text { past combinations of } C_{i} \text { and } C_{j} \text {. }\end{array}$ \\
\hline
\end{tabular}

Note.

${ }^{\mathrm{a}} W=1 /\left(d_{i} d_{j}\right)$ is a normalization factor. $d_{i}$ stands for the number of times a article, journal, or subject category $i$ has been cited in the given time interval.

subject categories are based on percentages. Combinations of journals or subject categories constitute a certain percentage of all combinations in the respective category. The anticipation score for journals (AJR) and subject categories (ASC) is the difference between these future and past percentages. Positive values mean that the combinations become more popular in the future, while negative values indicate that the popularity decreases.

The anticipation scores defined above give a sense of what anticipation means in the present context: instead of being an index of typicality, it attempts to grasp the direction of change in a shifting field. However, to calculate the score one has to know the future citation rates for combinations at $T_{1}$. It is not useful for prognosis. Of course this all depends on the time window one chooses for $T_{1}$. If someone wants to evaluate the future impact of recently published articles, one can set this time window to 0 . This gives a simplified index also shown in Table 2. This alternative index is the ratio of past and present combination frequencies. One is added to the denominator, so no division with zero occurs. The nominator is always at least one, because one paper in the data set must make this combination in the present to consider it in our calculation. That index can be interpreted as an indicator if a certain combination takes a fresh momentum. Note that no weighting is added to this index. It is not necessary because the denominator (past frequencies) already contains information on the popularity of the combined elements. Using the past frequencies instead of the citation impact of the combined elements has a different meaning. While the latter deflates the given score for popular journals or citations, the first only deflates their scores if they were already used together. Instead of assuming a "blind variation," the alternative measures encapsulate the field-specific usage pattern change.

\section{Results}

Raw citation counts were converted to a binary variable. Several studies employ this strategy, and focus on high-impact papers, and use a binary variable that indicates the top $5 \%$ of citation impact (Schilling \& Green 2011; Uzzi et al. 2013; Lee et al. 2015). Many independent variables remain skewed after log transformation (Figures 1-4). CIT mean, AJR, CIT (alt.) is close to being normally distributed. Several distributions have irregular shapes. JR mean is almost bimodal. An interesting observation that we can make just based on the histograms is that most articles cite novel paper combinations. From this perspective, novelty-seeking behavior is the dominant strategy. On average, $67 \%$ of a paper's citations are new article combinations (more precisely, these combinations are new in the sense that they did not occur in the past seven years). Both ASC and AJR are also heavier on the positive side, which suggests that the disciplinary diversity increased in astrophysics and astronomy in that time. Indeed the number of new journal pairs increased by $29 \%$, and the new subject category pairs by $39 \%$ in the given time period. The growth rate of cited paper combinations was $19 \%$. It is also worth mentioning that the range of ASC is higher than AJR. Journal associations appear to be more conservative than sub-discipline boundaries.

To be able to present the association of citation impact and these other variables concisely and in a similar way for comparison, all independent variables are analyzed by their percentiles. This means that in the following figures and calculations, the probability of a hit paper is plotted along the percentiles of the independent variables, which avoids the analytical drawbacks of these non-normal distributions, and also allows detection of nonlinear associations.

Surprisingly, the novelty measures are not in line with previous findings (Figure 5). The average of combinatorial novelty decreases citations in the future. This rate is not completely linear for subject category pairs. There are several explanations for these findings. While most studies found a positive relationship between the central tendency of novelty and citation impact (Schilling \& Green 2011; Leahey \& Moody 2014; Kaplan \& Vakili 2015), the study by Uzzi et al. (2013) also found a negative association. Current studies show mixed findings about the success of publications combining sub-disciplines in terms of receiving citations (Lariviere et al. 2015; Yegros-Yegros et al. 2015). Boyack \& Klavans (2014) showed that although combinatorial novelty generally brings success to articles, the magnitude of these effects depends on the disciplinary affiliation of the articles. In our case, as we can see later, novelty on the level of subject category pairs varies with the sub-fields of astronomy and astrophysics, and it is the citation impact of the sub-fields that 

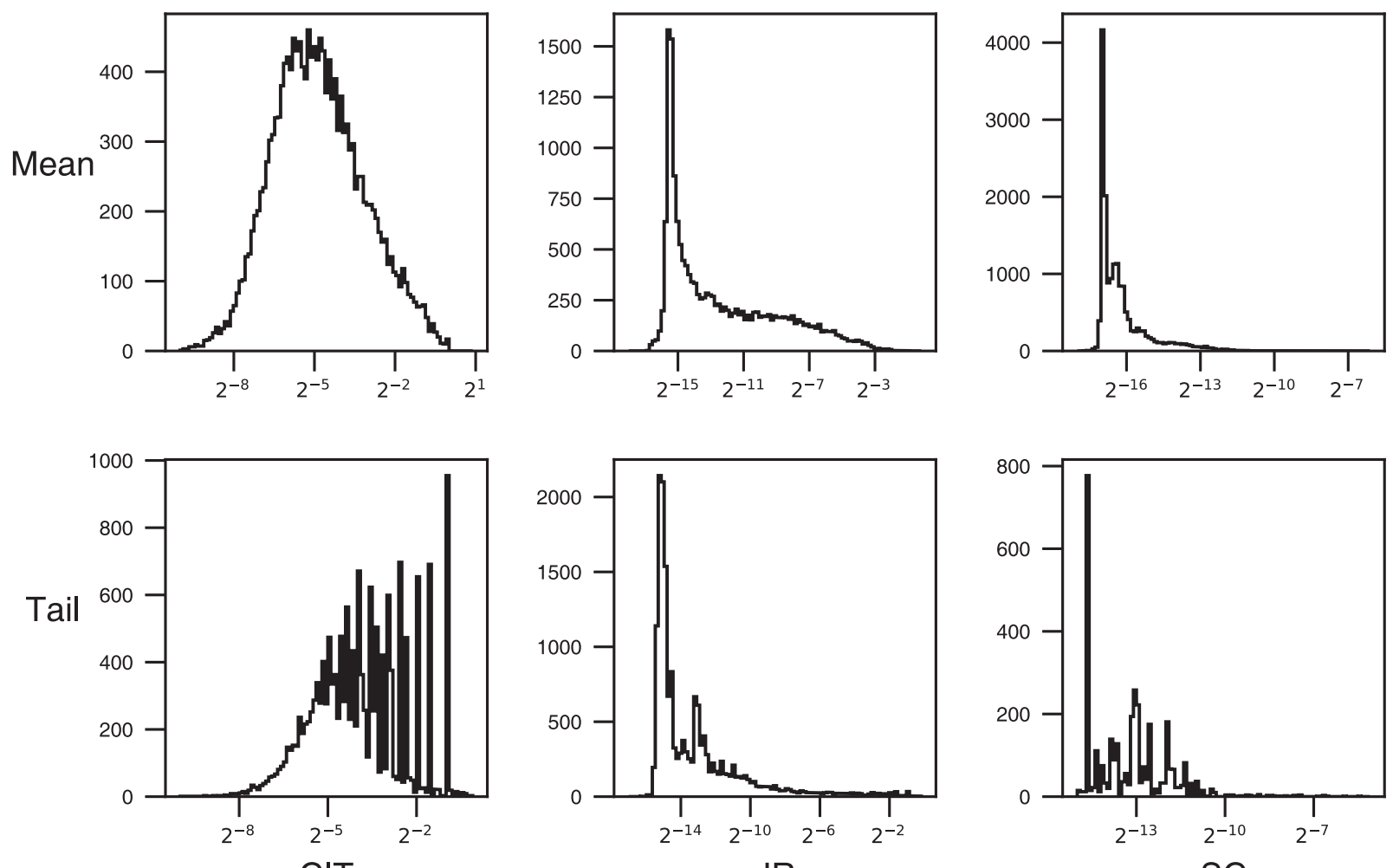

Figure 1. Distribution of novelty indexes. The figure shows the distribution of the three novelty scores (by columns), and their respective means and 90th percentiles (by rows).

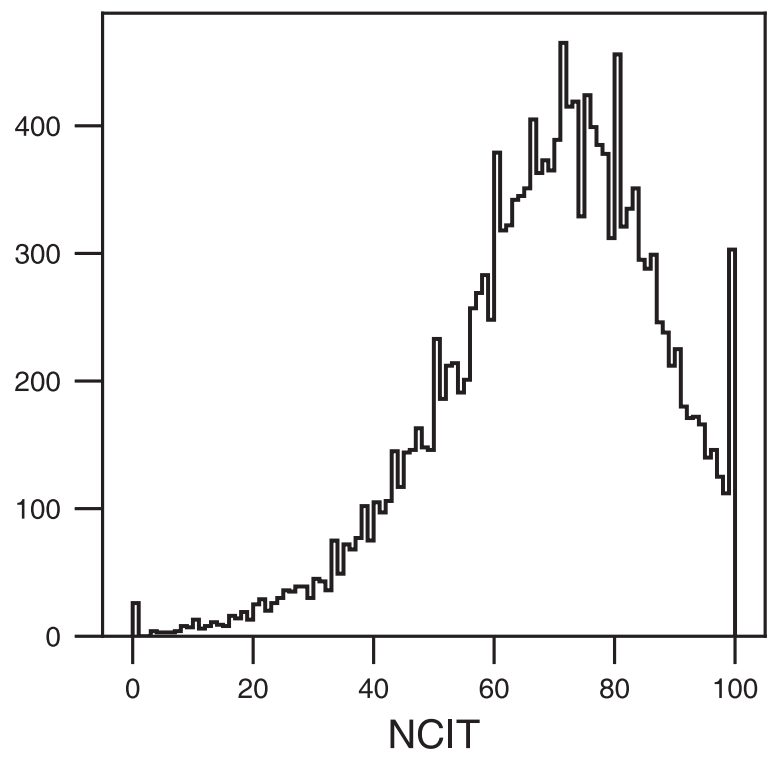

Figure 2. Distribution of the percentage of new combinations of research documents.

explains the citation outcomes of an article. A more general explanation of this finding is that the normalization weight deflates the novelty scores of those combinations that are taking off in the sampled years, which would penalize research that sets up a trend by gaining fresh citations in the sampled years. The negative effect especially seems to be strong for CIT, for which such a novelty index has not been constructed yet.

Taking a look at the effect of the percentage of new article combinations (NCIT, Figure 6), one can see that it has an inverted $U$ shape: the most successful referencing strategy is to combine new papers, albeit doing so in moderation. This corroborates the observation of Uzzi et al. (2013), and the hypotheses of Schilling \& Green (2011), and Leahey \& Moody (2014). All these studies assumed, for various theoretical reasons, that producing atypical combinations has a threshold at which the return on citation impact diminishes. These studies used different methodologies, and no study used NCIT or a similar index as a measure of novelty, but the current findings are in harmony with their expectations.

Turning to anticipation scores (Figures 7 and 8) first one should notice how crucial it is to cite early papers that will gain popularity later (ACIT). This has a strong association with citation impact. The top three percentiles of ACIT have at least a $\sim 15 \%$ chance of containing high-impact articles. CIT (alt.) also have a positive association with citation impact. However, knowing the future co-citation frequencies (like in ACIT for the following 7 years) clearly has a better explanatory power over the future popularity of articles.

The behavior of the indexes that are based on paper citations is markedly different from the ones based on journal and subject category pairs. While the first type can be approximated with a monotonically increasing function (see details below), that is not true for the latter type. It is difficult to describe these associations. They are remarkably similar to each other across the two types of measures: AJR is similar to JR (alt.), and ASC is similar to SC (alt.). In the case of journal-based measures the highest percentiles of the anticipation scores clearly yield more citations, while the subject-category-based measures show a fast decrease to the baseline 5\% at around the 80th percentile. This decrease happens earlier for CIT (alt.). This visual approach also 

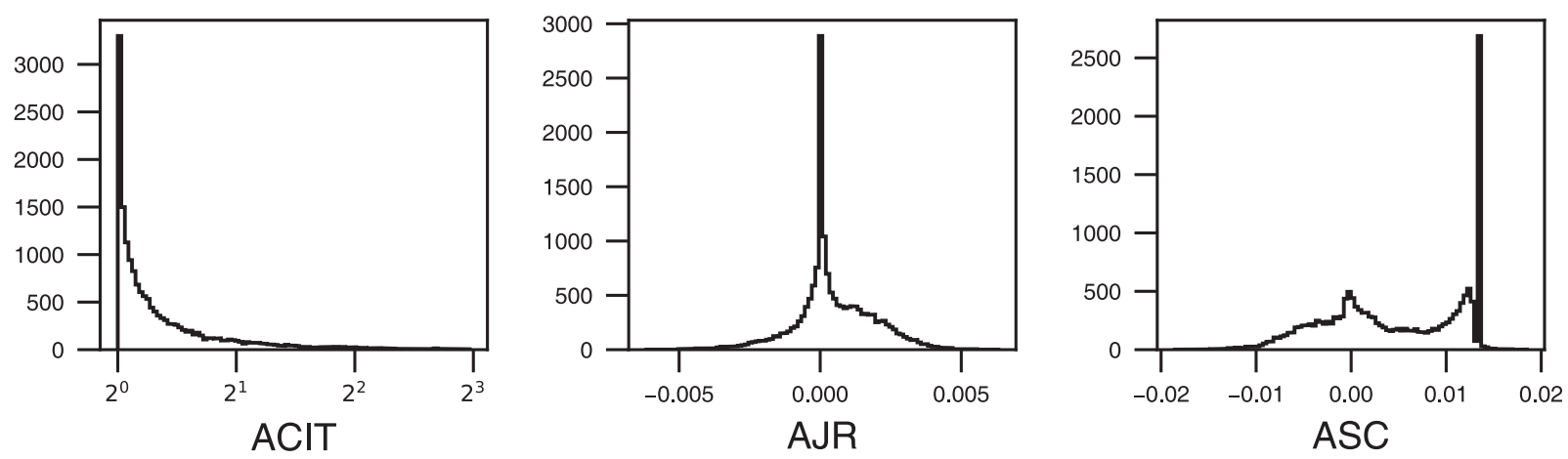

Figure 3. Distribution of the mean of anticipation scores. One is added to the ACIT score to fit on the log scale.
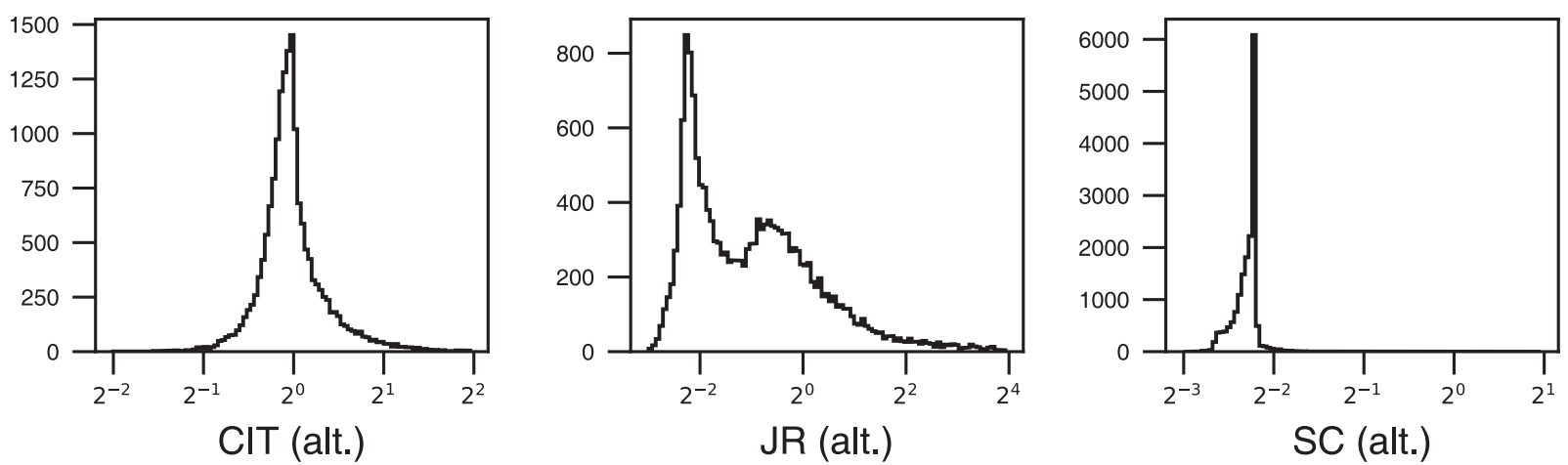

Figure 4. Distribution of the mean of "alternative" novelty scores, based on the concept of anticipation.

Table 3

Fitted Model Statistics and Mutual Information for Novelty and Anticipation Indexes

\begin{tabular}{|c|c|c|c|c|}
\hline & Model & " Bivariate Model, Residual d. ${ }^{\text {a }}$ & "Hierarchical Model, Residual d. ${ }^{\mathrm{b}}$ & Mutual Information $^{\mathrm{c}}$ \\
\hline$\overline{\text { NULL }}$ & $\operatorname{Logit}(\hat{Y})=B_{0}$ & 6672.3 & 6668.7 & $\ldots$ \\
\hline CIT (a.) & $\operatorname{Logit}(\hat{Y})=B_{0}+B_{1} X+B_{2} X^{2}$ & 6291.7 & 6288.4 & 0.021 \\
\hline JR (a.) & $\operatorname{Logit}(\hat{Y})=B_{0}+B_{1} X+B_{2} X^{2}$ & 6628.6 & 6276.7 & 0.009 \\
\hline NULL & $\operatorname{Logit}(\hat{Y})=B_{0}$ & 6672.3 & 6672.3 & $\cdots$ \\
\hline ACIT & $\operatorname{Logit}(\hat{Y})=B_{0}+B_{1} X+B_{2} X^{2}$ & 6155.8 & 6155.8 & 0.026 \\
\hline
\end{tabular}

Notes.

${ }^{a}$ Residual deviance of bivariate logistic regressions. All models are significant at the $>3 \sigma$ confidence level.

${ }^{\mathrm{b}}$ Residual deviance of hierarchical logistic regressions. Variables for the novelty and anticipation scores are entered in the order shown in the table. All models are significant at the $>3 \sigma$ confidence level.

${ }^{\mathrm{c}}$ MI stands for mutual information in bits.

has its limitations. Percentiles are sometimes ambiguous. One can observe in Figures 5, 7, and 8 that the subject-categorybased scores have several percentiles around the 80th percentiles that have the same percentile value.

These results show that while the anticipation of citation trends clearly increases the chance of publishing a high-impact paper, if the shift involves the crossing of sub-disciplines or research field boundaries, the reception of rewards has a more complex mechanism. Later we will see that this phenomenon can be partly explained by the sub-disciplinary structure of astronomy and astrophysics.

Given these counterintuitive findings about CIT, JR, and SC, and to stay more focused, for the remaining of the article only the anticipation scores will be investigated further.
Table 3 and Figures 9 and 10 show the results of an attempt to fit statistical models on the anticipation/novelty scores. The primary reason to construct statistical models is to compare the magnitudes, and possibly to describe the relationship better. Polynomial logistic regressions have been fitted to the data, using the $R$ package $g n m .{ }^{1}$ Figures 9 and 10 and plot the logit of the odds of hit papers by the percentiles of the scores. One can see that the models generally fit poorly to the data, especially for the journal and subject category measures. The tests of the residual changes, however, are all statistically significant (Table 3 ). The author tried to fit the best functions up to degree 4 polynomials to the data. Using

1 https://cran.r-project.org/web/packages/gnm/ 

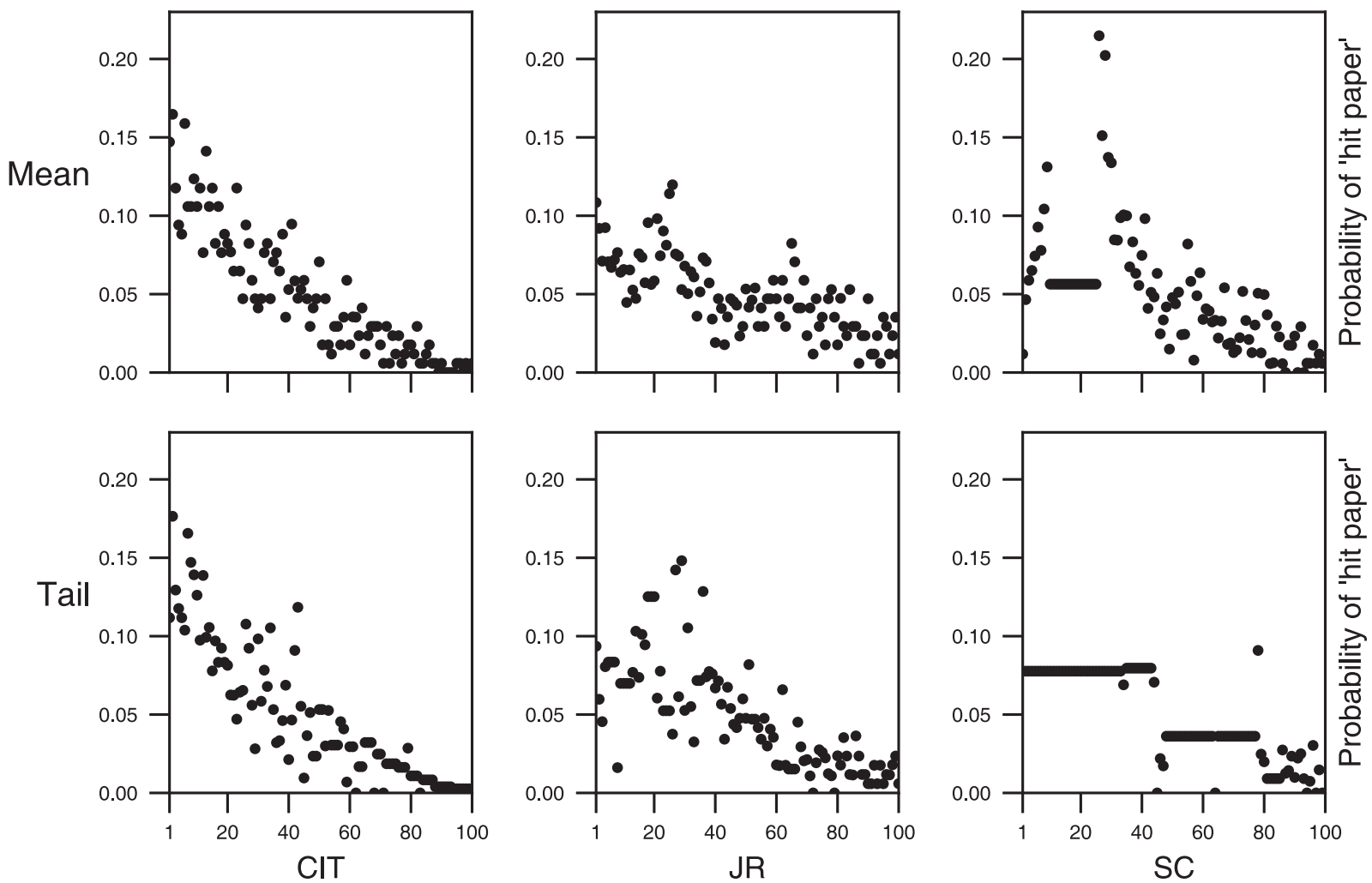

Figure 5. "Hit paper" probability by the means and 90th percentiles of the publications' novelty scores (by column). The x-axis is the percentiles of the respective novelty score statistic (mean or 90th percentile, row-wise).

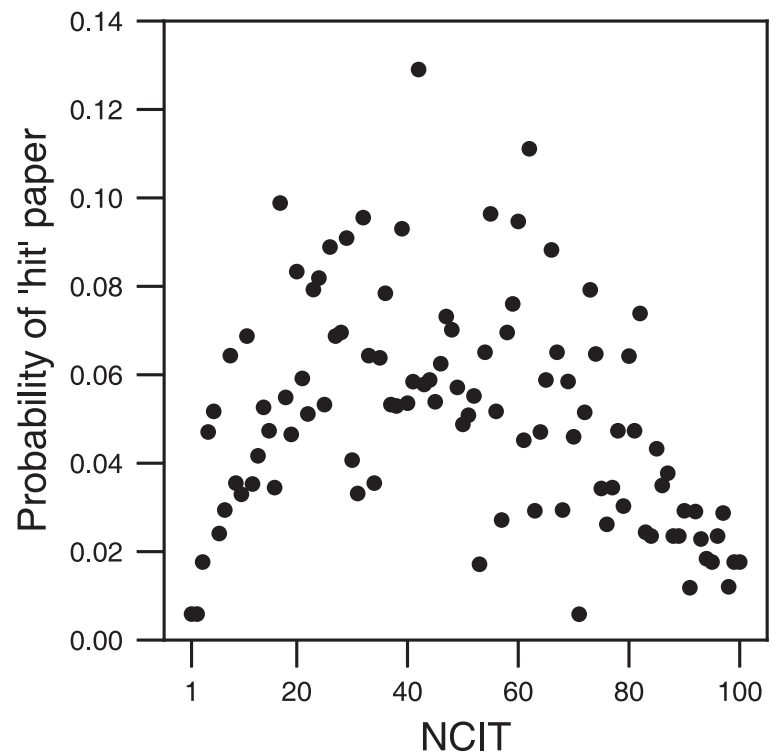

Figure 6. "Hit paper" probability by percentage of new combinations.

other types of functions in the analysis was difficult because of the frequent non-convergence of the maximum-likelihood estimators. Table 3 shows both the bivariate association of the independent variables and the dependent variable, and the hierarchical model where the independent variables were entered in the following order: citation-, journal-, subjectcategory-based metrics. Both the subject category scores and the journal-based scores are statistically significant when they are entered in the hierarchical models. This suggests that the three levels of aggregation explain different aspects of how to gain citation impact. However, we have to be careful to make this conclusion because of the poor fit.

In order to somewhat remedy the problems with the logistic models, mutual information scores are also reported in Table 3. Mutual information was fitted to the binary variables "hit paper," and the percentiles of the scores. This measure does not enforce any particular form of the relationship. According to these results, citation-based measures have the best explanatory power. The alternative $\mathrm{SC}$ index is better in that regard than the alternative JR index, while AJR is slightly better than ASC. However, this ordering is not stable; see the results for the 2007 data set in the Appendix (Table 6). Although one cannot make a clear judgment about the magnitudes of these relationships, the more linear character makes the paper-based magnitudes more desirable for prognosis.

Finally, we should investigate a little more closely the distribution of these indexes within the discipline. Figures 11 and 12 show citation maps of the most important journals that published astronomy and astrophysics articles in 2003 and 2007. Tables 4 and 5 show the indexes for influential journals for these two years. Below, I provide an interpretation of the visualization, but since this is an exploratory tool, the reader may find other approaches to the presented evidence. These maps do not contain all the journals that published relevant articles determined by the sampling method of this paper. The selection criteria for the drawings and the tables was the total citations each journal received in the period under study. Only the top $\sim 70$ journals were included for the drawing, and the tables show information for the top 36 journals. The size of the nodes in the figures is proportional to this value. The networks were drawn and manipulated in Gephi (Bastian et al. 2009). 

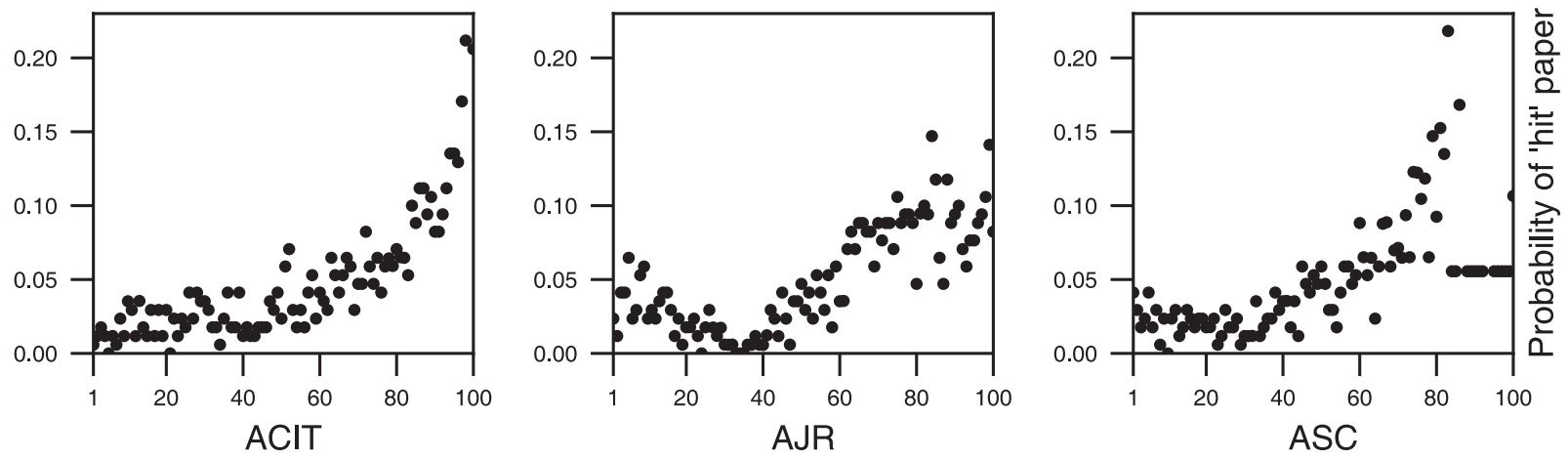

Figure 7. "Hit paper" probability by the percentiles of the publications' anticipation scores.
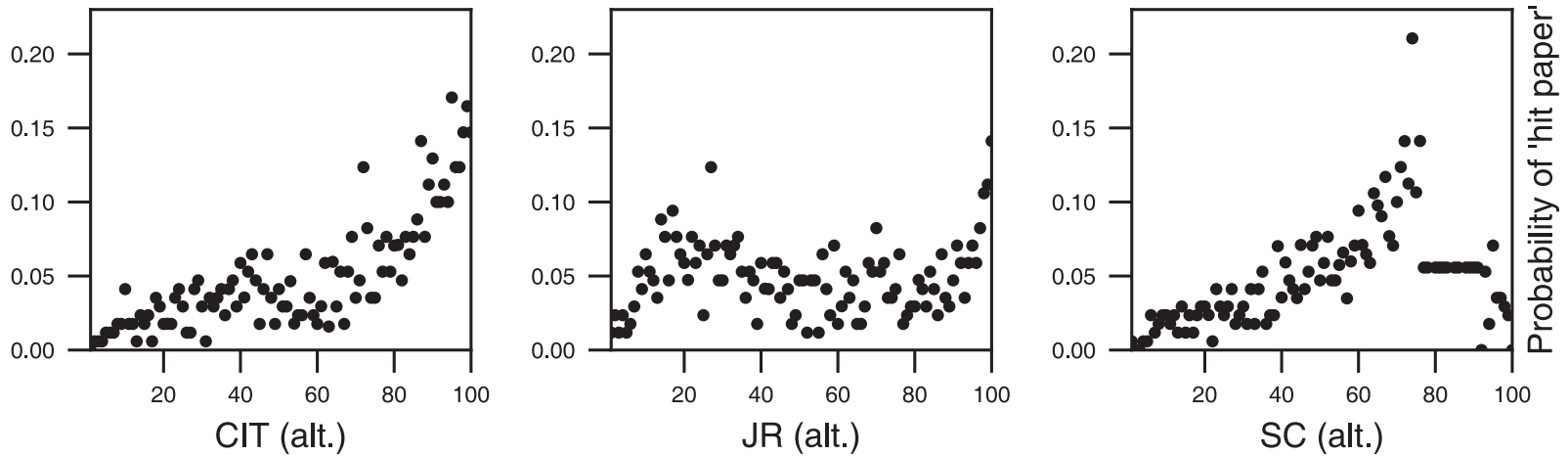

Figure 8. "Hit paper" probability by percentiles of alternative definitions of novelty scores.

Table 4

Novelty and Anticipation Indexes by Core Journals in 2003

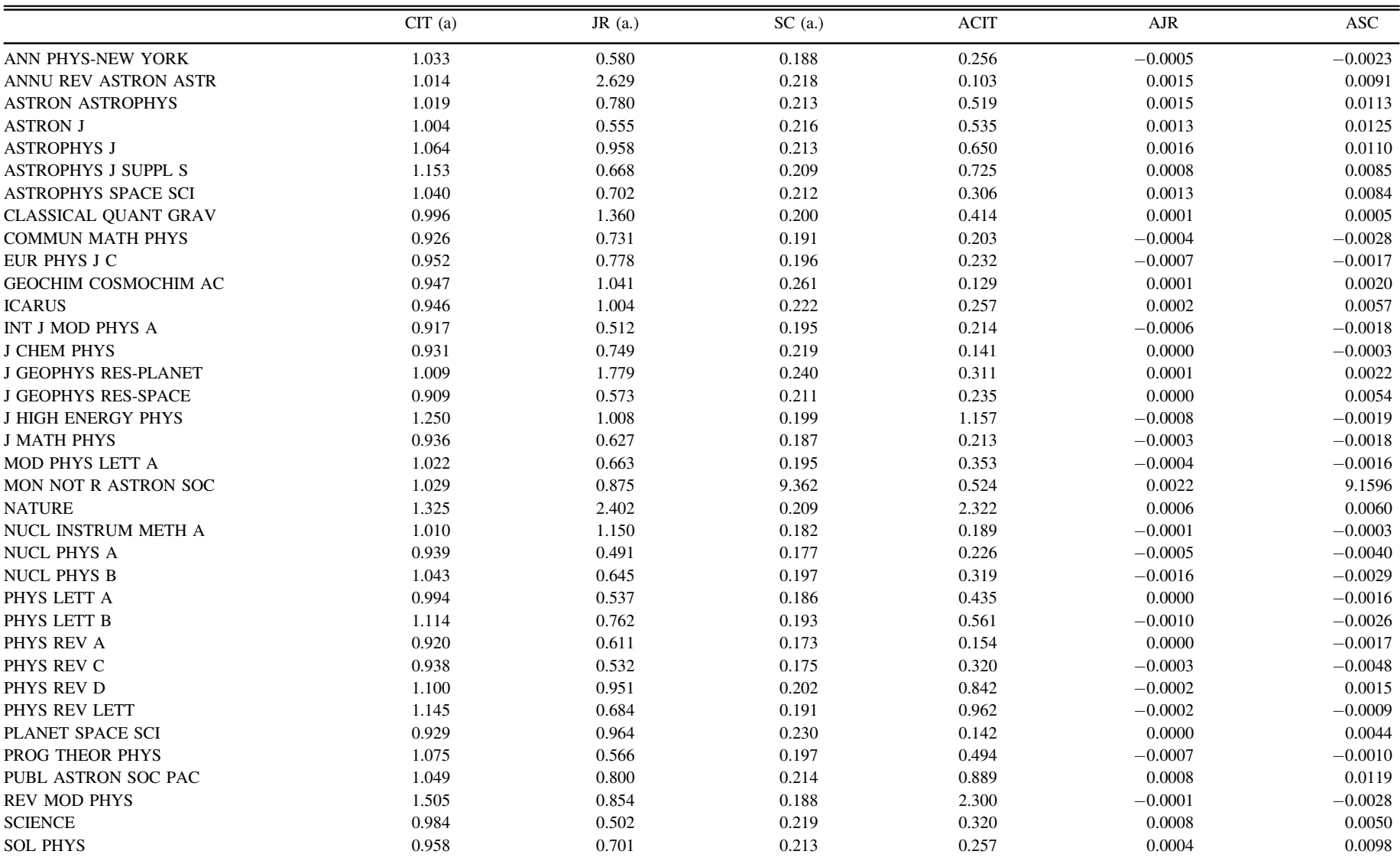




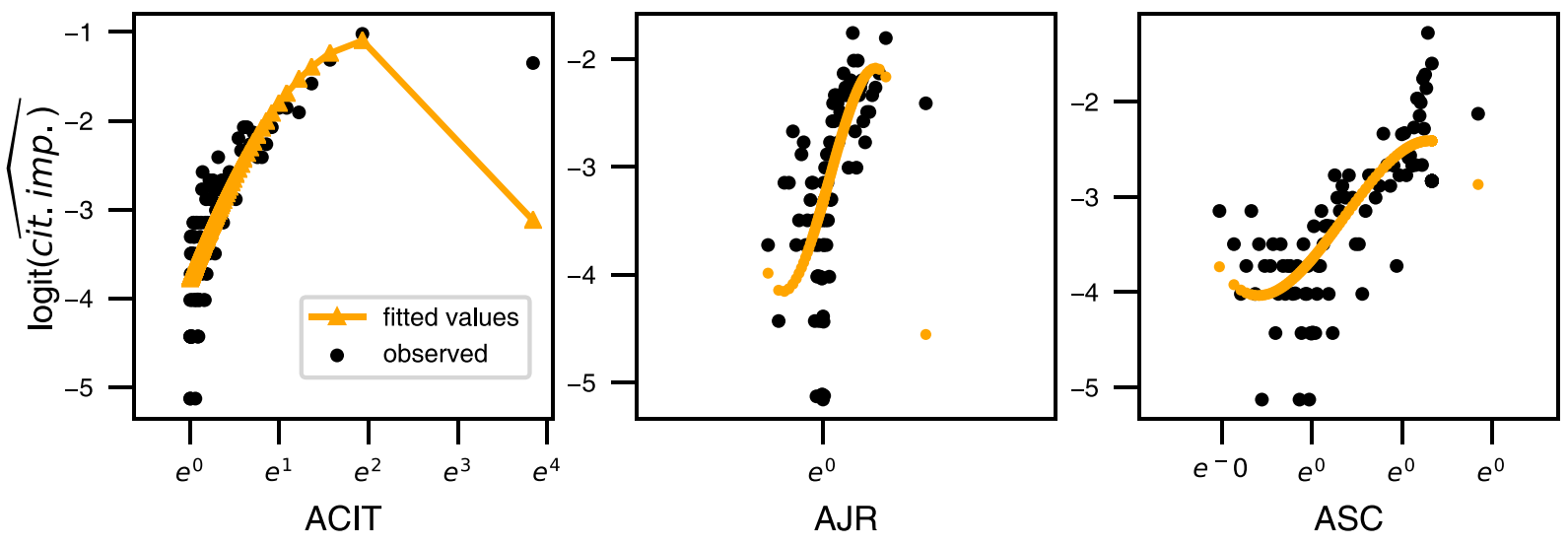

Figure 9. Observed and fitted log of the odds of publishing a hit paper as a function of anticipation scores.

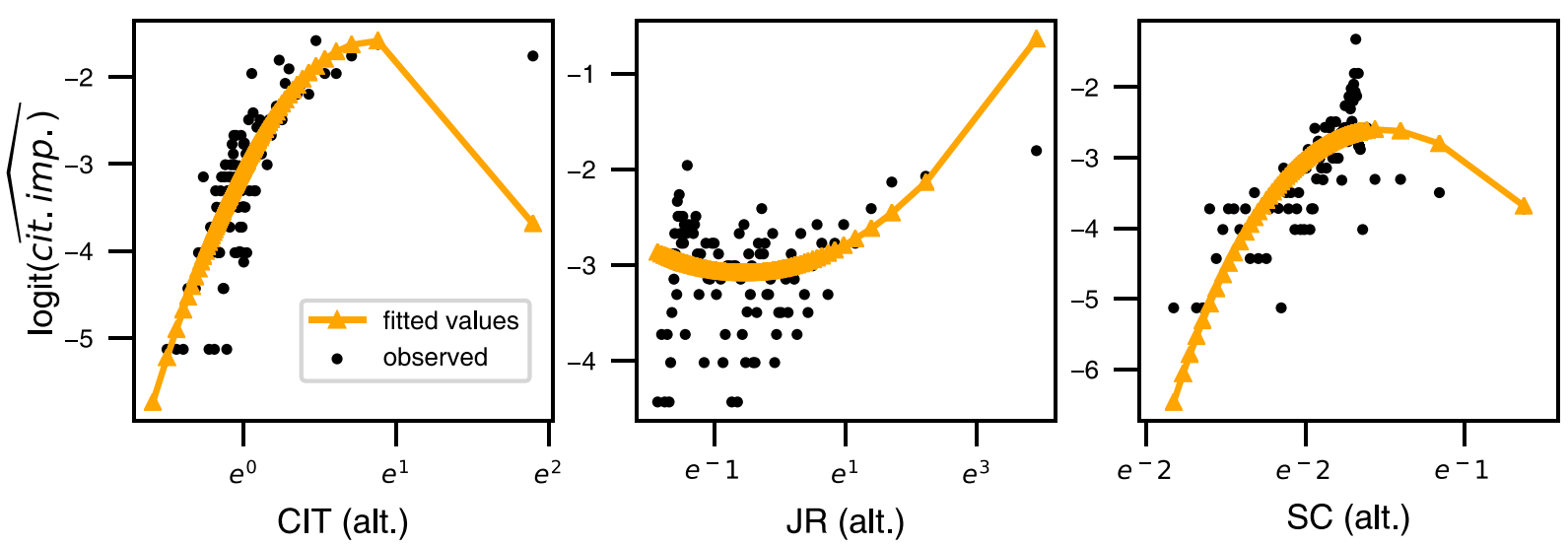

Figure 10. Observed and fitted log of the odds of publishing a hit paper as a function of alternative novelty indexes.

The positions of the journals reflect their relative distance to each other given their propensity to cite one another. The nodes were manually repositioned to make the journal names nonoverlapping. There is a clear division on the map between physics, and astronomy and astrophysics journals. This reflects the division between theoretical and observational astronomy. The center is occupied by the journals published by the American Astronomical Society, and other generalist astronomy journals. This means that they connect the two fields. In the upper regions one can see the planetary sciences with spectroscopy and physical chemistry. On the physics side one can clearly see a division between certain physics journals that are central for astronomy, and on the other hand specialized nuclear and mathematical physics journals, which are further away from astronomy. One can see that the above described regions changed in this relatively short time interval from 2003 to 2007 , most importantly the theoretical and observational sides moved closer to each other.

How does this morphology correlate with the indexes? Several observations can be made that support and clarify the earlier observations about our metrics. Consider the citationbased metrics. Larger nodes in the center have higher scores, and they set up trends for citation combinations. The Annual Review of Astronomy and Astrophysics is interesting in that regard. It has the highest impact factor in the discipline. Reviews have a specific communicative function in a discipline, so it is informative to see the scores this journal receives. While its mean CIT (alt.) score is moderately high, the ACIT score is low. This makes sense, because being a review journal its attention is fixated on the well-established results (combinations) of the past. The journal-based metrics have a different distribution over the regions. Astronomy and planetary science show more activity in this dimension in 2003, and the JR (alt.) scores are higher toward the planetary science edge. This difference between the two main regions is even more pronounced on the longer timescale of AJR. The theoretical physics journals have a conservative, stable journal combination pair structure, while most of the reconfiguration shifts toward the center of astronomy and astrophysics. However, this difference is not observable in 2007, when the general physics journals moved closer to the "astronomy pole." The Annual Review of Astronomy and Astrophysics again display special behavior on the map: it is very responsive to the shifts in journal co-citation pairs. The subject-category-based indexes look similar to the journal-based indexes: theoretical physics is more stable than the rest of the map in 2003. One can see the peak of shorter term activity (SC 


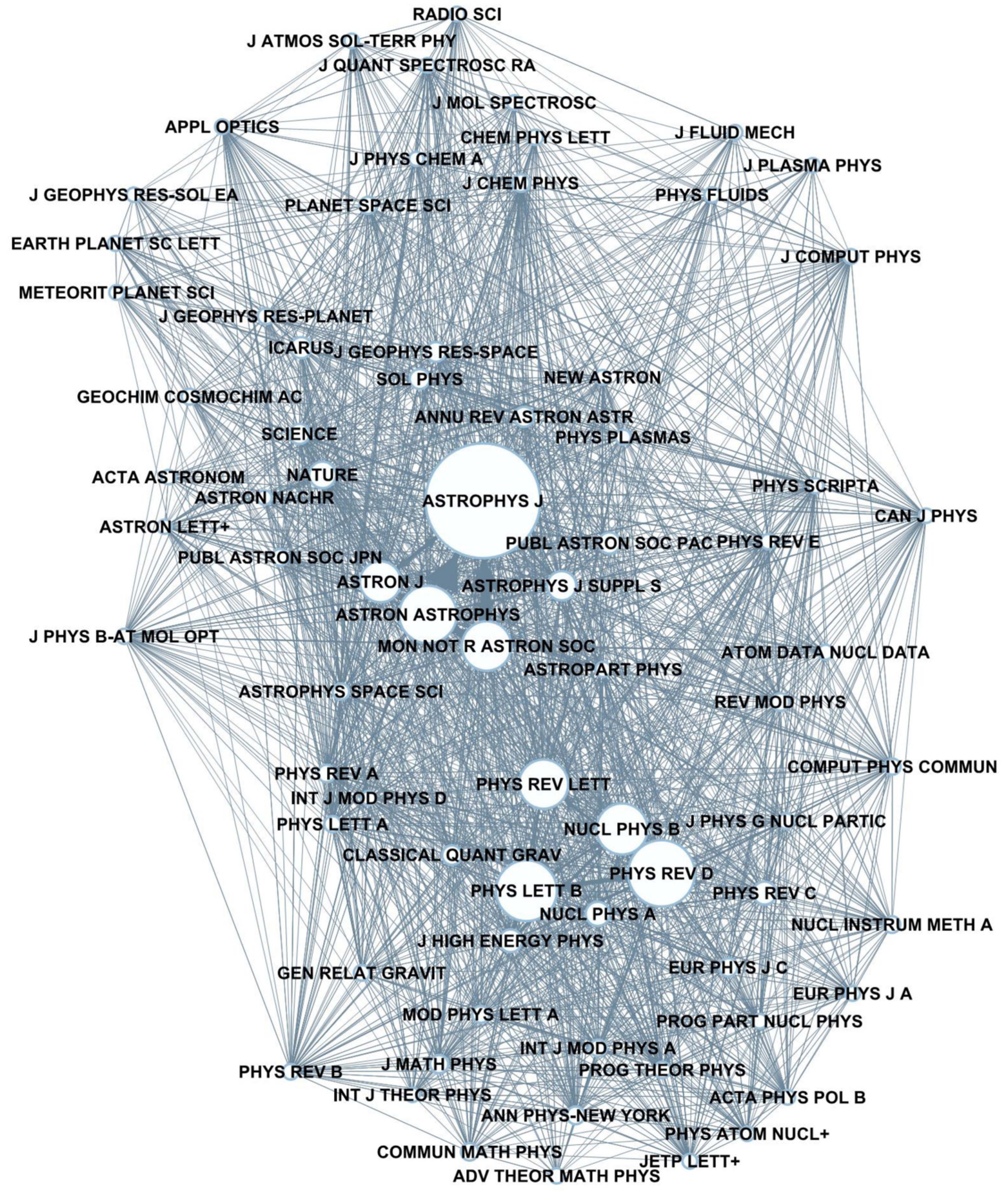

Figure 11. Citation networks of core astronomy and astrophysics journals in 2003.

(alt.)) around the planetary sciences and physical chemistry. However, this peak on the longer time span (ASC) shifts to the center of the discipline.

To summarize these observations, the theoretical physics region is more conservative in terms of relying on stable journal and subject category combinations in the long run, while the interdisciplinary activity happens on the observational astronomy side. Again, we can see that the indexes grasp different aspects of the changes in disciplinary practices. While the selection of focus on the literature is happening in the core between theoretical physics and observational astronomy, as one would expect, the interdisciplinary activity is more on the observational side. This "division of labor" between the sub-fields of the regions can explain the nonlinear association of citation impact and the journal- and subject-category-based indexes (Figures 8 and 9): more specialized sub-fields and journals with lower citation rates initiate the reconfiguration. This clearly holds for JR (alt.) (compare Figures 9 and 12). The behavior of the Annual Review of Astronomy and Astrophysics suggests that the selection of papers happens at a different timescale than the selection of new 


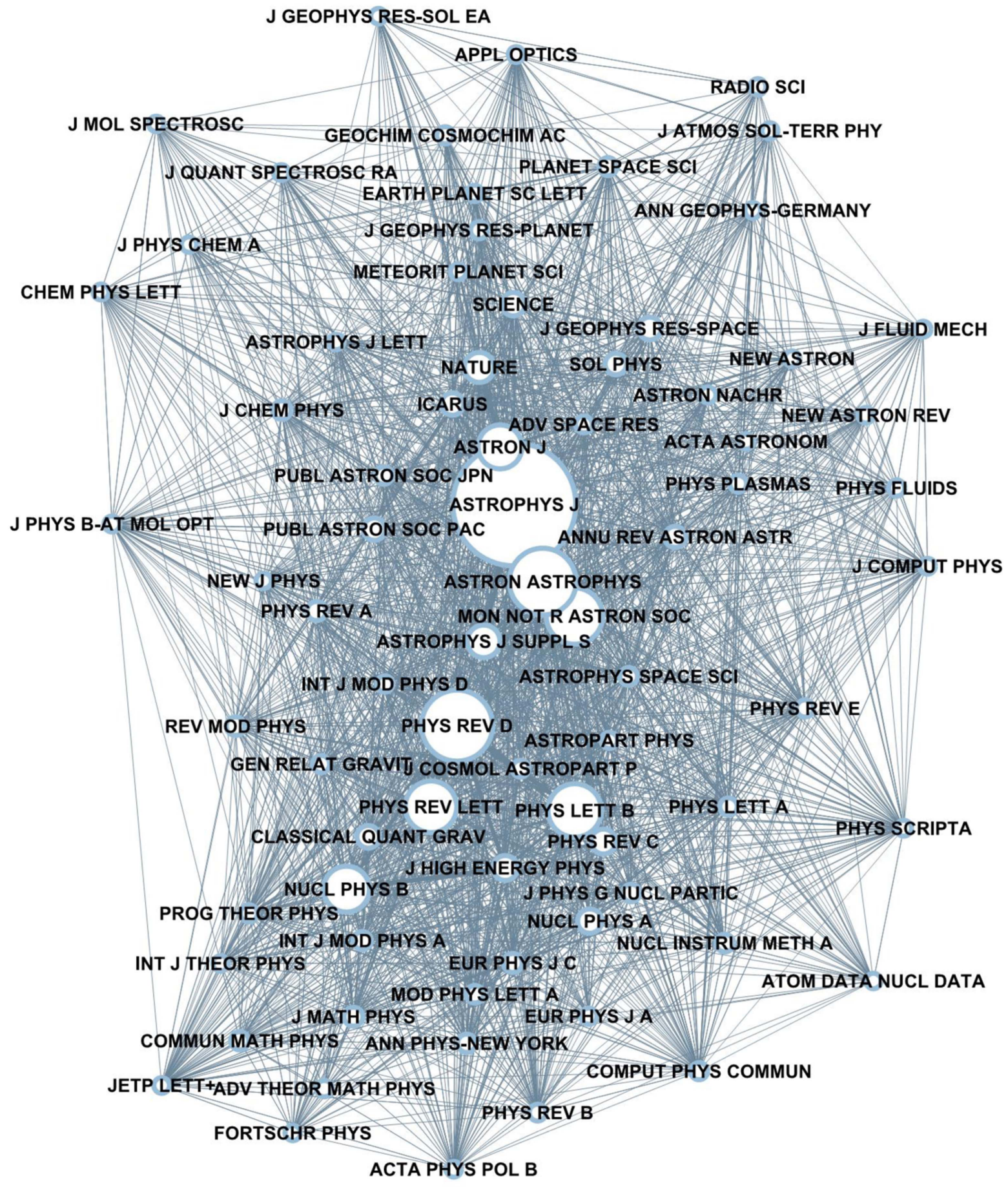

Figure 12. Citation networks of core astronomy and astrophysics journals in 2007.

institutional boundaries represented by typical journal and subject category pairs. While the Annual Review of Astronomy and Astrophysics reacts to the latter by being at the 65th-90th percentiles with its journal- and subject-category-based scores, its citation-based scores are around the 45th-65th percentiles.

\section{Discussion}

The studied indexes of combinatorial novelty have a tangible effect on citation impact in astronomy and astrophysics. The index based on the article level anticipation score (CIT alt.) is shown to be a more straightforward tool for predictions of future disseminations of research findings than higher-level predictors, because of its linear association with citation impact. However, the magnitudes of these associations are rather weak. The measures poorly discriminate "hit papers" from the rest. The highest percentage of hit papers in the highest percentiles of the CIT (alt.) indexes are around $17 \%-18 \%$, while the baseline is $5 \%$. This is very far from finding $100 \%$ of high-impact papers by utilizing such indexes derived from reference lists. At this point it is not possible for scientometrics to fully predict the potential of a paper or a 
Table 5

Novelty and Anticipation Indexes by Core Journals in 2007

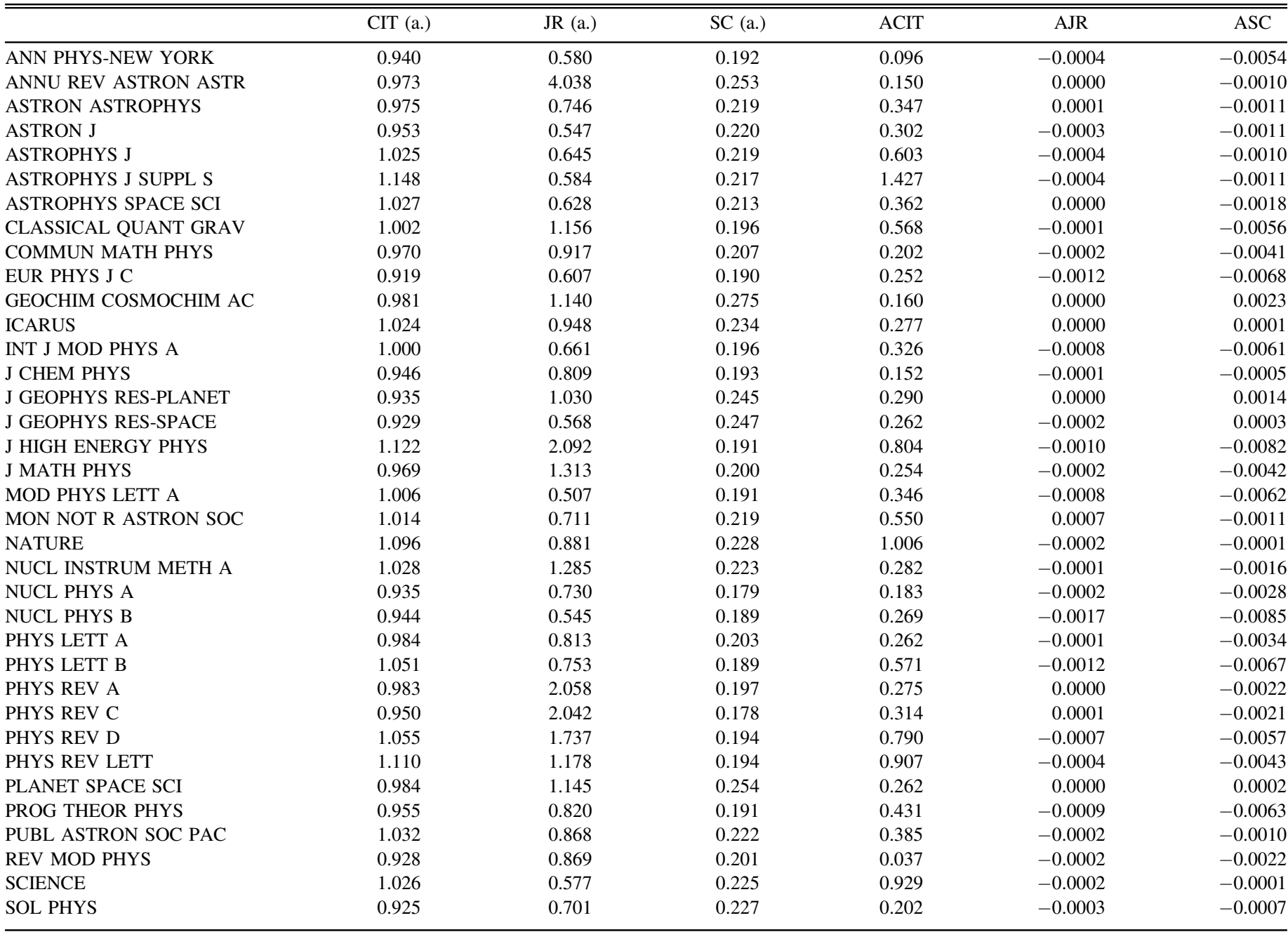

Table 6

Fitted Model Statistics and Mutual Information for Novelty and Anticipation Indexes for 2007

\begin{tabular}{lllcc}
\hline \hline & Model & Bivariate Model, Residual d. $^{\text {a }}$ & Hierarchical Model, Residual d. $^{\text {b }}$ & Mutual Information $^{c}$ \\
\hline NULL & Logit $(\hat{Y})=B_{0}$ & 7628.7 & 7624.5 & 7272.7 \\
CIT (a.) & Logit $(\hat{Y})=B_{0}+B_{1} X+B_{2} X^{2}$ & 7277.74 & 7270.1 & 0.017 \\
JR (a.) & $\operatorname{Logit}(\hat{Y})=B_{0}+B_{1} X+B_{x} X^{2}+B_{3} X^{3}$ & 7619.937 & 7122.7 & 0.007 \\
SC (a.) & $\operatorname{Logit}(\hat{Y})=B_{0}+B_{1} X+B_{2} X^{2}$ & 7488.188 & 7628.73 & 0.021 \\
NULL & $\operatorname{Logit}(\hat{Y})=B_{0}$ & 7628.73 & 7033.9 & 0.026 \\
ACIT & $\operatorname{Logit}(\hat{Y})=B_{0}+B_{1} X+B_{2} X^{2}$ & 7033.885 & 7012.6 & 0.011 \\
AJR & $\operatorname{Logit}(\hat{Y})=B_{0}+B_{1} X+B_{2} X^{2}$ & 7590.33 & 6884.6 & 0.016 \\
ASC & $\operatorname{Logit}(\hat{Y})=B_{0}+B_{1} X+B_{x} X^{2}+B_{3} X^{3}$ & 7493.774 & & \\
\hline
\end{tabular}

Notes.

${ }^{a}$ Residual deviance of bivariate logistic regressions. All models are significant at the $>3 \sigma$ confidence level.

${ }^{\mathrm{b}}$ Residual deviance of hierarchical logistic regressions. Variables for the novelty and anticipation scores are entered in the order shown in the table. All models are significant at the $>3 \sigma$ confidence level, except the addition of JR.

${ }^{\mathrm{c}}$ MI stands for mutual information in bits.

scientist, or to predict the fate of research programs (Clauset et al. 2017). Altogether, metrics that are devised to detect trend changes-referred to here as anticipation scores-have good potential to be used in future studies. The proposed scores have a simple rationale, showing whether a given paper is citing combinations that are gaining popularity at the moment. In other words, this illustrates which combination pairs are getting fresh attention. Future studies may reveal whether the studied associations with citation impact are stable in fields other than astronomy and astrophysics. 
This study presents several theoretically relevant findings about how the dissemination of innovation is shaped by institutional factors. It also provides methodological reflections on the better utilization of these indexes. Furthermore, article level scores-which have been neglected by previous research on the topic-have substantial potential. Conventional novelty measures actually have a negative effect on citation impact. Given the contradictory findings about this relationship in the literature, the result is not novel. Creating more new paper combinations (NCIT) is advantageous, but only until a certain threshold, beyond which it becomes a bad strategy. This is not a completely new finding. A previous study by Yegros-Yegros et al. (2015) found similar associations between impact and performing interdisciplinary research, although their methodology was different from the one applied here (Porter et al. 2007). Moreover, other studies (Schilling \& Green 2011; Leahey \& Moody 2014) hypothesized this inverted $U$ shape, but did not find it with their data and methods. Their reasoning is that spanning boundaries of specialized knowledge is considered to be innovative and beneficial, because one can rely on a greater scope and diversity of information, but it is also risky in several respects. For example, it is hard to master several specializations, and the audience can be resistant or unprepared to consume exported knowledge.

The aggregation level differences of the metrics are strong. Not only is the association pattern with citation impact markedly more complex at the journal and subject category level than on the paper level, but the journal citation maps also reveal journal clusters in the discipline as being responsible for trend changes at different levels. In short, theoretical physics is less responsive to institutional changes in the field that are taking off in the investigated time period. In light of this data, astrophysics and astronomy experience some shift in focus in the early 2000s. Heidler (2011) investigated the changing disciplinary relations of astrophysics, and argued that the discovery of the accelerating expansion of the universe in $1998 / 1999$, and the discovery that dark energy and dark matter constitute $95 \%$ of the universe, induced the increased influence of high-energy physics and the importance of cosmology, especially in the United States. Indeed, one can observe by comparing the journal citation maps of 2003 (Figure 11) and 2007 (Figure 12) that astronomy/astrophysics and the pure physics journals moved closer to each other. The growing importance of exoplanet research and the experimental demonstration of gravitational waves will lead to further new and exciting research. Studying the processes of how these new findings gain footing is an interesting topic for future scientometric studies.

I am thankful for the help of Erin Leahey, Philip Rosenfield, Gretchen Stahlman, and the anonymous reviewer. I am also thankful for the University of Arizona HPC services.

\section{Appendix}

These figures show the same analysis for the year 2007 (Figures 13-16). The curves on these figures are the same as
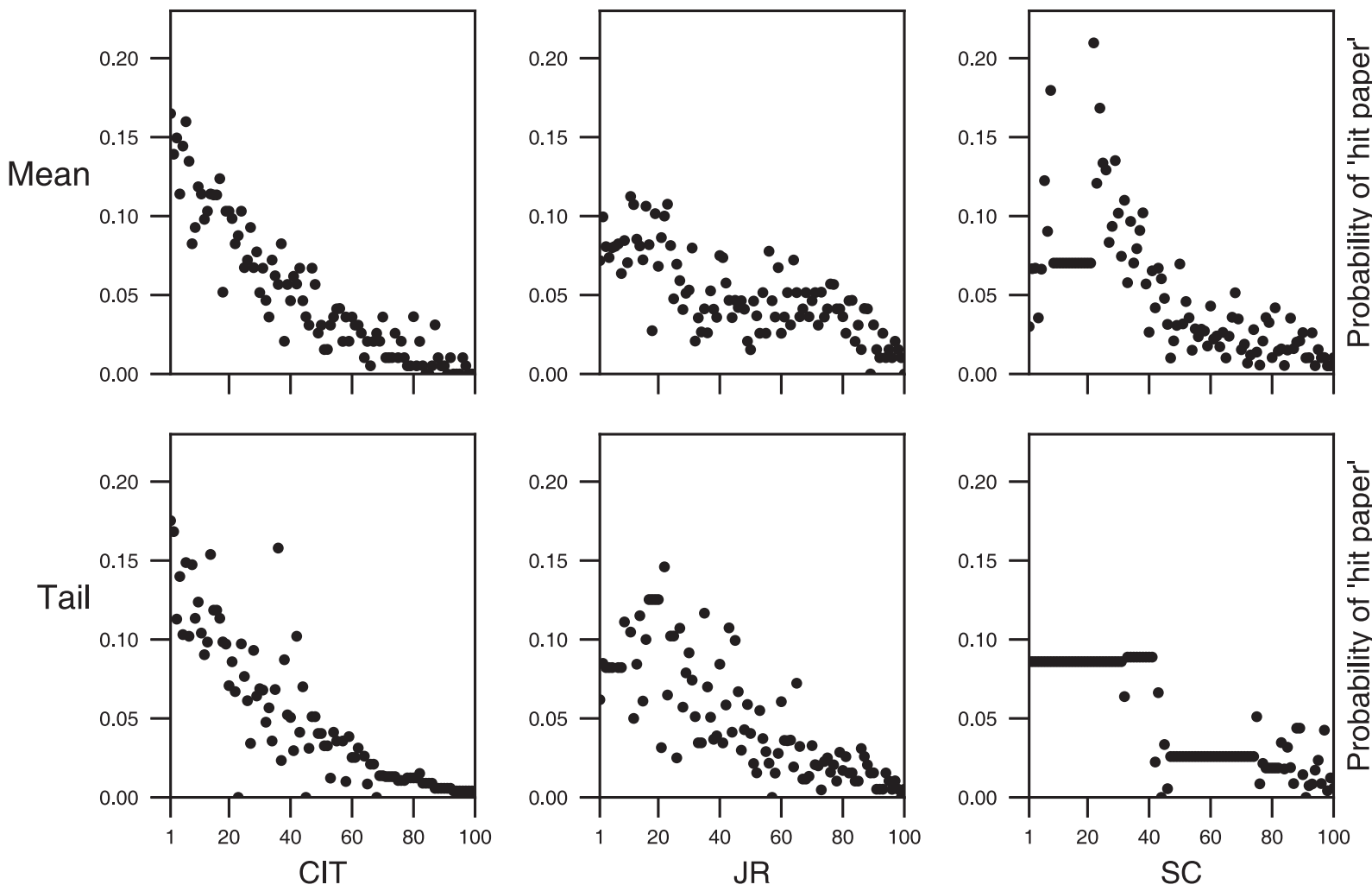

Figure 13. "Hit paper" probability and the means and 90th percentiles of the publications' novelty scores (by column) in 2007. 


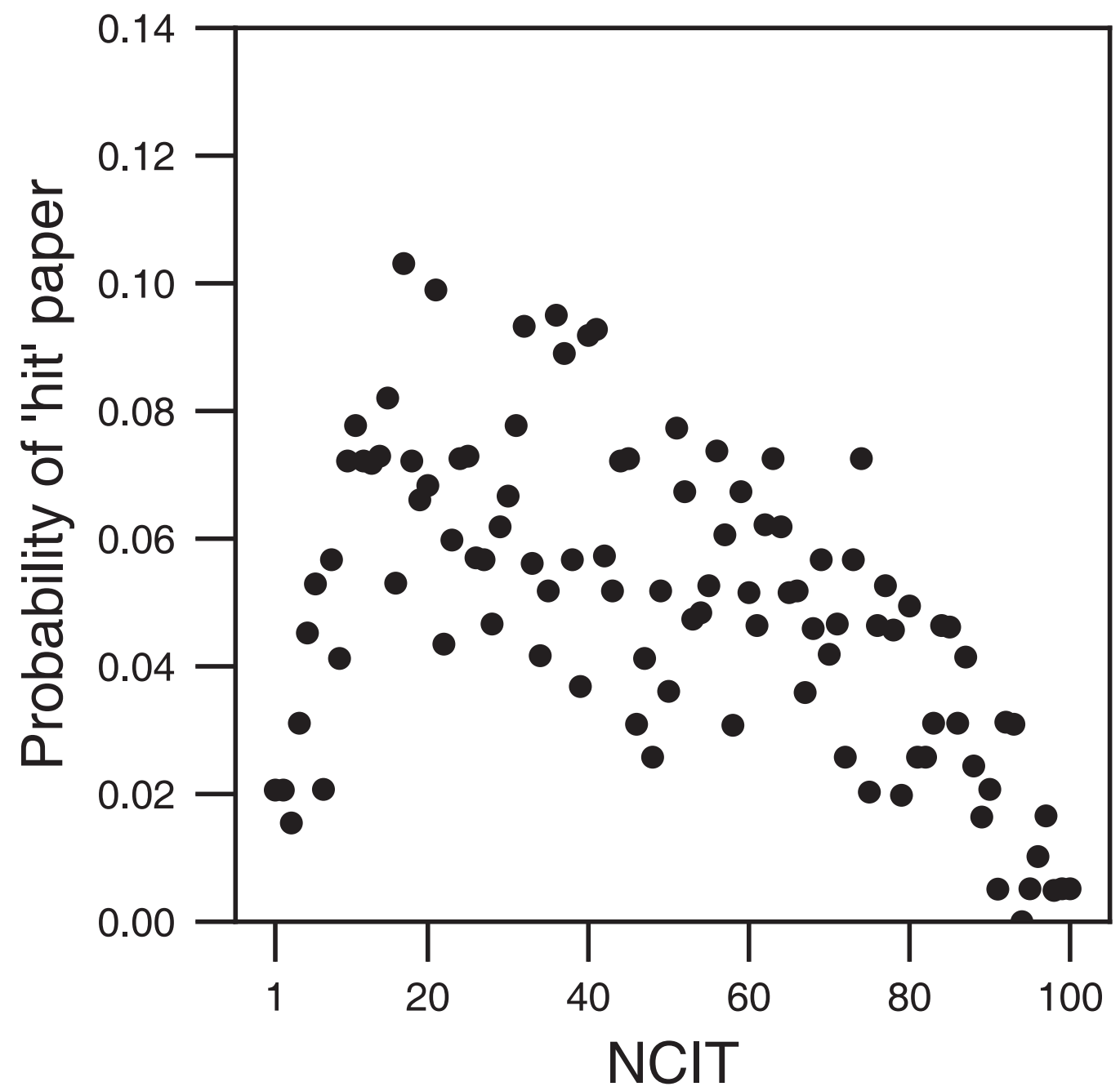

Figure 14. "Hit paper" probability by percentage of new combinations in 2007.
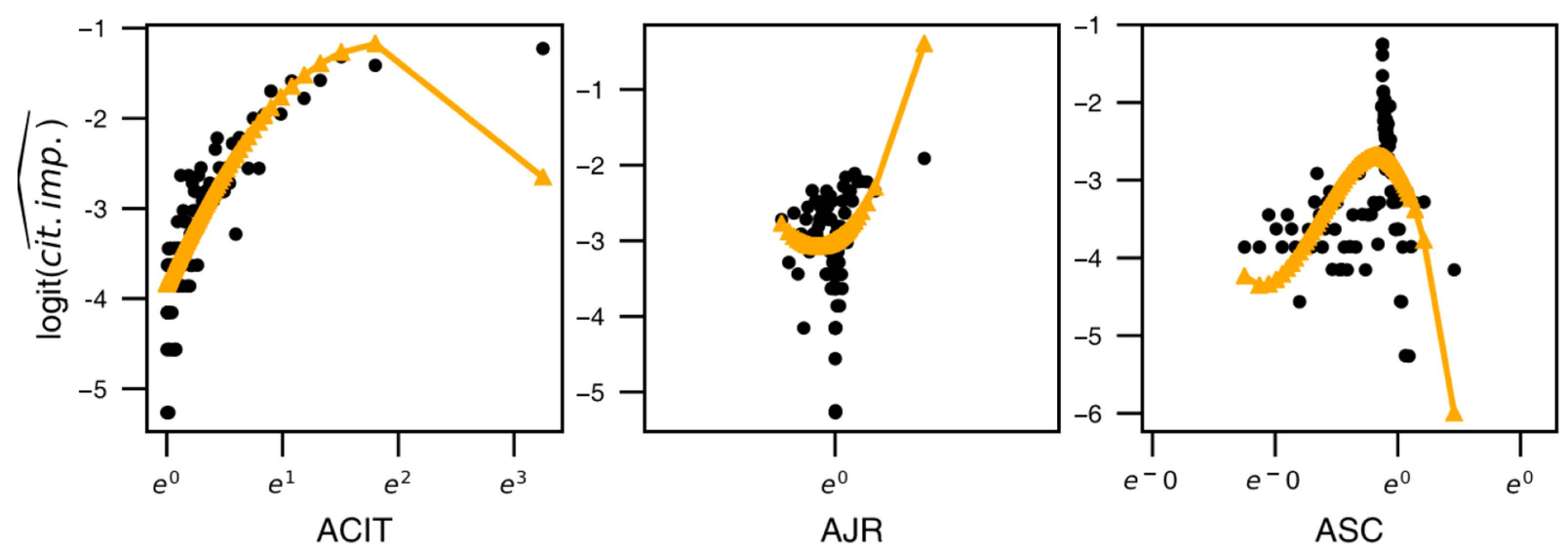

Figure 15. Observed and fitted log of the odds of publishing a hit paper as a function of anticipation scores in 2007. 

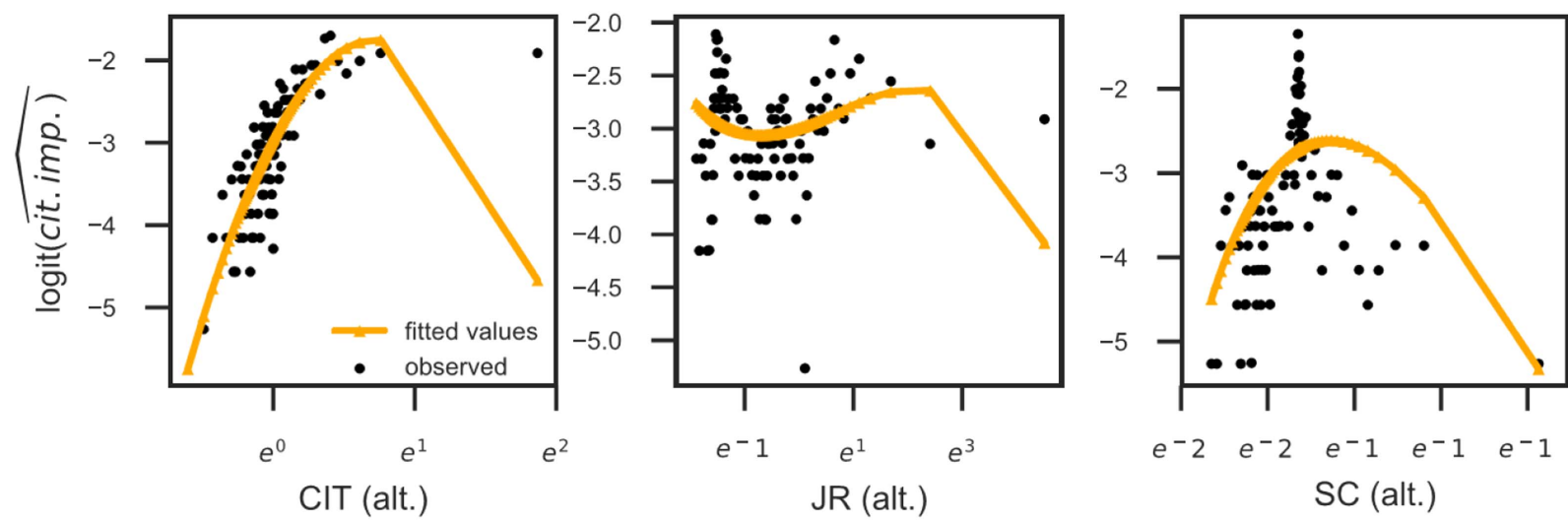

Figure 16. Observed and fitted log of the odds of publishing a hit paper as a function of alternative novelty indexes in 2007.

those in the main text, and they serve as a reference. The results are very similar across the three years.

\section{References}

An, X., \& Huang, J. X. 2017, Journal of the Association for Information Science and Technology, 68, 2620

Bastian, M., Heymann, S., \& Jacomy, M. 2009, in Int. AAAI Conference on Weblogs and Social Media, ed. E. Adar (Menlo Park, CA: AAAI Press), 361

Boyack, K. W., \& Klavans, R. 2014, in Proc. Science and Technology Indicators Conf., ed. E. Noyons (Leiden: Universiteit Leiden), 64

Carbonell, J., \& Goldstein, J. 1998, in Proc. 21st ACM SIGIR Conf., ed. B. Croft et al. (New York, NY: ACM), 335

Clarke, C. L., Kolla, M., Cormack, G. V., et al. 2008, in Proc. 31st ACM SIGIR Conf., ed. T. Chua \& M. Leong (New York, NY: ACM), 659

Clauset, A., Larremore, D. B., \& Sinatra, R. 2017, Sci, 355, 477

Evans, J. A. 2010, American Journal of Sociology, 116, 389

Fleming, L. 2001, Management Science, 47, 117

Heidler, R. 2011, Minerva, 49, 461

Kaplan, S., \& Vakili, K. 2015, Strategic Management Journal, 36, 1435

Kurtz, M. J., \& Henneken, E. A. 2015, Journal of the Association for Information Science and Technology, 68, 695

Lariviere, V., Haustein, S., \& Boerner, K. 2015, PloSO, 10, e0122565
Leahey, E., \& Moody, J. 2014, Social Currents, 1, 228

Lee, Y., Walsh, J. P., \& Wang, J. 2015, Research Policy, 44, 684

Leydesdorff, L. 1998, Scim, 43, 5

Leydesdorff, L. 2001, The Challenge of Scientometrics: The Development, Measurement, and Self-organization of Scientific Communications (Irvine, CA Universal-Publishers)

Poincaré, H. 1910, The Monist, 20, 321

Porter, A. L., Cohen, A. S., Roessner, J. D., et al. 2007, Scim, 72, 117

Rafols, I., \& Meyer, M. 2010, Scim, 82, 263

Savino, T., Messeni Petruzzelli, A., \& Albino, V. 2017, International Journal of Management Reviews, 19, 54

Schilling, M. A., \& Green, E. 2011, Research Policy, 40, 1321

Schumpeter, J. A. 1934, The Theory of Economic Development (Cambridge, MA: Harvard)

Sinatra, R., Deville, P., Szell, M., et al. 2015, NatPh, 11, 791

Small, H., Boyack, K. W., \& Klavans, R. 2014, Research Policy, 43, 1450

Small, H. G. 1978, SSS, 8, 327

Thagard, P. 2012, in Psychology of Science: Implicit and Explicit Processes, ed. R. Proctor \& E. J. Capaldi (Oxford: Oxford Univ. Press), 389

Trapido, D. 2015, Research Policy, 44, 1488

Uzzi, B., Mukherjee, S., Stringer, M., et al. 2013, Sci, 342, 468

Yegros-Yegros, A., Rafols, I., \& D’Este, P. 2015, PloSO, 10, e0135095 Youn, H., Strumsky, D., Bettencourt, L. M. A., et al. 2015, Journal of the Royal Society Interface, 12, 20150272 